\title{
Characterization of throat microbial flora in smokers with or without COPD
}

This article was published in the following Dove Press journal:

International Journal of COPD

4 July 2017

Number of times this article has been viewed

\author{
Wenqi Diao* \\ Ning Shen* \\ Yipeng Du \\ Ke Qian \\ Bei He \\ Department of Respiratory Medicine, \\ Peking University Third Hospital, \\ Beijing, China \\ *These authors contributed equally \\ to this work
}

\begin{abstract}
The study aimed to determine the relationship between throat microbiome and COPD. Sixty-five Chinese males ( $n=20$, smokers without COPD; $n=45$ smokers with COPD) were included. Nonmetric multidimensional scaling indicated differences of microbiome between COPD and controls, but no difference was observed between COPD patients with differing degrees of lung function or disease severity. Rarefaction analyses suggested that operational taxonomic units (OTUs, species-level) richness decreased in COPD. The dominant taxa between COPD and controls were similar, but the proportions of taxonomic distribution were different. The dominant phyla were Bacteroidetes, Proteobacteria, Firmicutes and Fusobacteria. The dominant genera were Haemophilus, Leptotrichia, Porphyromonas, Fusobacterium, Veillonella, Streptococcus, Neisseria and Prevotella. Two dominant OTUs, otu3 (Veillonella_dispar) and otu4 (Streptococcus_unclassified), were identified. Otu3 and its father-level taxa, which were negatively correlated with predicted percent of forced expiratory volume in a second ( $\mathrm{FEV}_{1} \%$ pred), were increased in COPD. By contrast, otu 4 and its father-level taxa, which were positively correlated with $\mathrm{FEV}_{1}$ \% pred, were decreased in COPD. Otu4 also showed a slight potential as a COPD biomarker. To conclude, the throat microbiome was different between smokers with or without COPD, which is similar to findings from the lower respiratory tract. This study may strengthen our understanding of the relationship between microbiomes of different airway sites and COPD.
\end{abstract}

Keywords: microbiome, throat, chronic obstructive pulmonary disease

\section{Introduction}

COPD, a common inflammatory pulmonary disease that is characterized by incompletely reversible airflow limitation and progressive loss of alveolar spaces, results in a considerable mortality and economic burden. ${ }^{1}$ Bacterial infection or colonization is considered to be a key causative factor of the occurrence and progression of COPD. ${ }^{2}$ In the past, the relationship between COPD and pathogens was investigated in terms of culturedependent methods, ${ }^{3,4}$ but this method only identified limited bacterial types.

Recently, high-throughput microbiome strategies have been applied to the COPD field and have identified the microbial features of COPD patients. ${ }^{5,6}$ These studies predominantly focused on bacterial communities of lower airway using induced sputum or bronchoalveolar lavage fluid (BALF) samples. ${ }^{7-9}$ However, microbiome studies of throat samples are limited. ${ }^{10,11}$ Moreover, the goal of these studies was to identify differences of throat flora between COPD and asthma patients, ${ }^{10,11}$ restricting deep analyses for the throat microbiome of COPD.

To our knowledge, the relationship between oropharyngeal microbial communities and COPD progression has not been determined. Throat swab is easier to collect than induced sputum and is safer than BALF, indicating that it may have a potentially wider

\footnotetext{
Correspondence: Bei He

Department of Respiratory Medicine, Peking University Third Hospital, 49 North Garden Road, Haidian District, Beijing I0019I, China

$\mathrm{Tel}+8601082265210$

Fax +86010 82086810

Email puh3_hb@bjmu.edu.cn
}

(c) (i) (5) 2017 Diao et al. This work is published and licensed by Dove Medical Press Limited. The full terms of this license are available at https://www.dovepress.com/terms.php cc) and incorporate the Creative Commons Attribution - Non Commercial (unported, v3.0) License (http://creativecommons.org/licenses/by-nd/3.0/). By accessing the work you hereby accept the Terms. Non-commercial uses of the work are permitted without any further permission from Dove Medical Press Limited, provided the work is properly attributed. For permission for commercial use of this work, please see paragraphs 4.2 and 5 of our Terms (https://www.dovepress.com/terms.php). 
clinical application than other samples. Thus, the present study aimed to compare the microbial communities of the throat between smokers and COPD patients with different degrees of lung function or disease severity.

\section{Methods}

\section{Participants}

A total of 65 subjects ( $n=20$, smokers without COPD; $n=45$ smokers with COPD) were recruited in the clinic of Peking University Third Hospital from November 2015 to February 2016. The study was approved by the Peking University Institutional Review Board and was strictly carried out in terms of experimental protocols. Written informed consent was obtained from all participants. These patients have been previously diagnosed as COPD and followed up in our clinic, but in this enrollment, they were still required to conduct a spirometry test. The predicted percent of forced expiratory volume in a second $\left(\mathrm{FEV}_{1}\right.$ \%pred) and the ratio of residual capacity and total lung capacity (RV/TLC) were determined through a ventilator (SensorMedics, Yorba Linda, CA, USA). All participants reported that they had no special diet habit, no known periodontal disease, no systematic antibiotics in 3 months, and did not use antiseptic mouthwash, corticosteroids and bronchodilators within $12 \mathrm{~h}$ before sample collection. Long-standing drug information, age, body mass index (BMI) and smoking history were also acquired by self-report of subjects.

Patients with COPD were included based on the following criteria: 1) males of 40-80 years, 2) smoking index of greater than 10 pack-years, and 3 ) diagnosed as a clinically stable stage of COPD according to the Global Initiative on Obstructive Lung Disease (GOLD), ${ }^{12}$ with a post-bronchodilator ratio of $\mathrm{FEV}_{1}$ and forced vital capacity $<0.70$, without an exacerbation within 8 weeks. Selected patients were excluded based on the following: 1) diagnosed with severe hepatic, cardiovascular, mental or renal dysfunction, 2) diagnosed with asthma, pulmonary cystic fibrosis or active pulmonary tuberculosis in 3 months and 3) had received immunosuppressive medications. In terms of severity of lung function, COPD patients were divided into two subgroups: group $1\left(\mathrm{n}=24, \mathrm{FEV}_{1} \%\right.$ pred $\left.\geq 50 \%\right)$ and group $2\left(\mathrm{n}=21, \mathrm{FEV}_{1} \%\right.$ pred $\left.<50 \%\right)$. According to GOLD classification, ${ }^{12}$ COPD patients were separated into GOLD_A, GOLD_B, GOLD_C and GOLD_D. Male smokers without COPD were selected as a control group, whose lung function and baseline information were also collected.

\section{Sample collection}

Sampling was conducted carefully in case of saliva contamination from other surfaces (ie, tongue and teeth) through a tongue depressor. Only posterior oropharynx was sampled using sterile cotton swabs by an experienced technician. After swabbing, the swab tips were put into sterile container and stored at $-80^{\circ} \mathrm{C}$ until further DNA extraction.

\section{DNA extraction, amplification and sequencing}

Each collected swab was randomly allotted to a new identifier for blindness. Bacterial DNA was extracted from all samples using the Qiagen DNA Extraction kit (Qiagen, Hilden, Germany) according to the modified protocol developed by the laboratory of Gary B Huffnagle. ${ }^{13}$ Amplification and sequencing of throat samples were performed at BGI (Shenzhen, China) in terms of Roche 454-based sequencing protocols. ${ }^{14,15}$ Briefly, the V3-V5 regions of the gene encoding $16 \mathrm{~S}$ ribosomal RNA (rDNA) were amplified by polymerase chain reaction using the forward primer (5'-CCGTCAATTCMTTTGAGTTT-3') and the reverse primer (5'-ACTCCTACGGGAGGCAGCAG-3') with sample-specific barcodes. After purification of amplicons, sequencing was conducted through 454 platform (Roche Applied Science, Basel, Switzerland).

\section{Microbial analysis}

Raw sff. files were processed by Mothur software (v.1.38.1, http://www.mothur.org) as described previously. ${ }^{16}$ All sequences were aligned through a NAST-based sequence aligner to a custom reference sequence based on the SILVA alignment (v102). Processed sequences were classified by a Ribosomal Database Project training set. High-quality sequences were annotated to specific operational taxonomic units (OTUs) at a 3\% distance cutoff.

\section{Statistical analysis}

Nonmetric multidimensional scaling (NMDS) based on jclass distance and analysis of molecular variance (AMOVA) were used to determine differences in bacterial communities between groups. Rarefaction curves with repeated-measures analysis of variance (ANOVA) were used to detect differences of OTU richness in the different groups. The Shannon and inverse Simpson indexes were calculated by Mothur software. The proportion of taxonomic distribution was compared by Fisher's exact test. The relative abundance of specific taxa and OTUs between COPD and controls was compared by Mann-Whitney $U(\mathrm{M}-\mathrm{U})$ tests. False discovery rate (FDR) was used to correct multiple testing. Bacteria with the raw $P<0.05$ and the FDR $<0.2$ were viewed as prominent ones. Correlations of bacterial taxa and clinical information were analyzed by Spearman coefficient. Receiver operating characteristic (ROC) curve analysis was performed 
to evaluate bacterial potential as a COPD marker. Statistical analyses were performed by SPSS 19.0, R software 3.3.0, MedCalc 15.1 or GraphPad Prism 5.0.

\section{Results}

\section{Study participants}

Baseline information for the 65 male subjects ( $\mathrm{n}=20$, smokers without COPD; $n=45$, smokers with COPD) is summarized in Table 1. COPD patients were divided into two subgroups: group $1\left(\mathrm{n}=24, \mathrm{FEV}_{1} \%\right.$ pred $\left.\geq 50 \%\right)$ and group $2(\mathrm{n}=21$, $\mathrm{FEV}_{1} \%$ pred $<50 \%$ ). Age, BMI and smoking history were not statistically different between COPD and controls. Usage of long-acting inhaled bronchodilators was very similar, but that of inhaled corticosteroids (ICSs) was obviously different between group 1 and group 2. More details are presented in Table 1.

\section{Comparison of bacterial communities among controls, group I and group 2}

To compare bacterial communities among different groups, unsupervised NMDS was used for data visualization, and AMOVA was used to determine statistical differences, as shown in Figure 1. Figure 1A indicated a spatial separation between the COPD and the control groups $(P=0.018$, jclass distance matrix, AMOVA); Figure 1B illustrated that the centroids were close between group 1 and group $2(P=0.818$, jclass distance matrix, AMOVA); and Figure $1 \mathrm{C}$ presented that no shift was observed between different GOLD stages. The NMDS and AMOVA based on Bray-Curtis distance matrix also showed a similar result, as presented in Figure S1.

\section{Rarefaction and alpha diversity analyses among controls, group I and group 2}

Rarefaction curve showed that OTU richness of COPD was lower than that of controls $(P<0.05$, repeated-measures
ANOVA), as presented in Figure 2. However, the OTU richness was not different between group 1 and group 2 or between different GOLD stages, as shown in Figure S2A and $\mathrm{B}$, respectively. These implied that OTU richness of COPD patients was decreased compared with that of the controls and was not associated with severity of disease or lung function. Figure S3 shows a decreased trend of Shannon and Simpson indexes in COPD patients compared with those of the controls. Similarly, no alteration was observed in COPD patients with different lung functions.

\section{Taxonomic distribution between smokers with or without COPD}

Figure 3 shows that the dominant taxa were the same, but the taxonomic distribution was different between the COPD patients and controls $(P<0.05$, Fisher's exact test) from phylum to genus level. The dominant phyla $(>1 \%)$ were Bacteroidetes, Proteobacteria, Firmicutes and Fusobacteria. The dominant genera $(>1 \%)$ were Haemophilus, Leptotrichia, Porphyromonas, Fusobacterium, Veillonella, Streptococcus, Neisseria and Prevotella. More detailed information is illustrated in Figure 3.

\section{Prominent bacterial taxa between smokers with or without COPD}

To explain the differences of taxonomic distribution between the COPD and the control groups, all prominent bacteria, with a $P<0.05$ calculated by M-U test and FDR $<0.2$, are shown in Figure 4. More details are listed in Table S1. At the (A) class, (B) order, (C) family and (D) genus level, we separately identified three, three, six and eight prominent bacterial taxa. No significant bacteria were found in phylum level. Notably, two highly abundant genera, Streptococcus and Veillonella, and their corresponding classes (Bacilli,

Table I Baseline data of subjects in different groups

\begin{tabular}{|c|c|c|c|c|}
\hline \multirow[t]{2}{*}{ Variable } & \multirow[t]{2}{*}{ Control $\mathbf{N}=\mathbf{2 0}$} & \multicolumn{3}{|c|}{ Smokers with COPD $(\mathrm{N}=45)$} \\
\hline & & Total $(\mathbf{N}=45)$ & Group I $(\mathbf{N}=\mathbf{2 4})$ & Group $2(\mathrm{~N}=\mathbf{2} \mid)$ \\
\hline Age (years) & $58.7 \pm 6.4$ & $62.3 \pm 7.0$ & $61.2 \pm 6.0$ & $63.6 \pm 7.9$ \\
\hline BMI $\left(\mathrm{kg} / \mathrm{m}^{2}\right)$ & $25.1 \pm 2.9$ & $24.3 \pm 2.8$ & $25.0 \pm 1.9$ & $23.5 \pm 3.4$ \\
\hline Smoking index (pack-years) & $32.2 \pm 15.4$ & $36.4 \pm 17.4$ & $40.8 \pm 19.0$ & $30.9 \pm 13.6$ \\
\hline FEV,$\%$ pred & $100.4 \pm 12.3$ & $55.0 \pm 18.5 * *$ & $69.7 \pm 9.9$ & $38.2 \pm 9.4^{\#}$ \\
\hline RV/TLC \% & $35.5 \pm 8.9$ & $52.7 \pm 12.4 * *$ & $43.9 \pm 7.2$ & $63.8 \pm 7.8^{\# \#}$ \\
\hline Inhaled corticosteroid & - & $15(33.3 \%)$ & $3(12.5 \%)$ & $12(57.1 \%)^{\#}$ \\
\hline Inhaled bronchodilator & - & $42(93.3 \%)$ & $21(87.5 \%)$ & $21(100 \%)$ \\
\hline \multirow[t]{4}{*}{ GOLD classification } & - & A I 2 (26.7\%) & A $12(50 \%)$ & - \\
\hline & - & B I2 (26.7\%) & B I2 (50\%) & - \\
\hline & - & C $8(17.8 \%)$ & - & C $8(38.1 \%)$ \\
\hline & - & D I3 (28.9\%) & - & D I3 (6I.9\%) \\
\hline
\end{tabular}

Notes: Continuous variables are presented as mean \pm standard deviation, and categorical variables are shown as $\mathrm{n}(\%)$. $* \boldsymbol{P}<0.0 \mathrm{I}$ represented the comparison between COPD and control. ${ }^{\#} P<0.01$ represented the comparison between group I and group 2 ; - denoted that it was not applicable. COPD patients included two subgroups: group I (FEV,\%pred $\geq 50 \%)$ and group 2 ( $\mathrm{FEV}_{1} \%$ pred $\left.<50 \%\right)$.

Abbreviations: BMI, body mass index; GOLD, Global Initiative on Obstructive Lung Disease; FEV , forced expiratory volume in I s; RV, residual capacity; TLC, total lung capacity. 
A

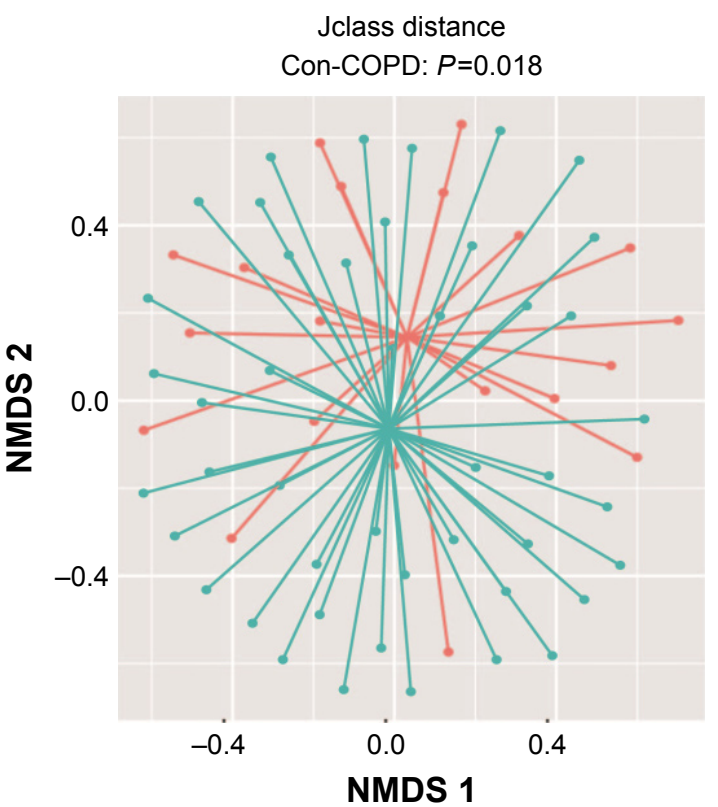

- Control - COPD
B

\section{Group 1 and group 2}

Jclass distance

Group 1 group 2: $P=0.818$

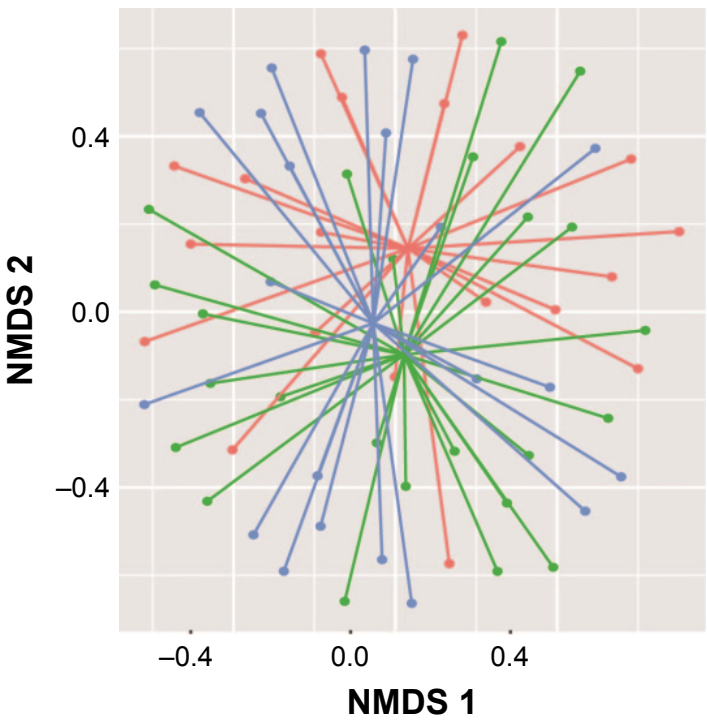

- Control

- Group 1 (FEV $\%$ pred $\geq 50 \%)$

- Group 2 (FEV $\%$ pred $<50 \%$ )

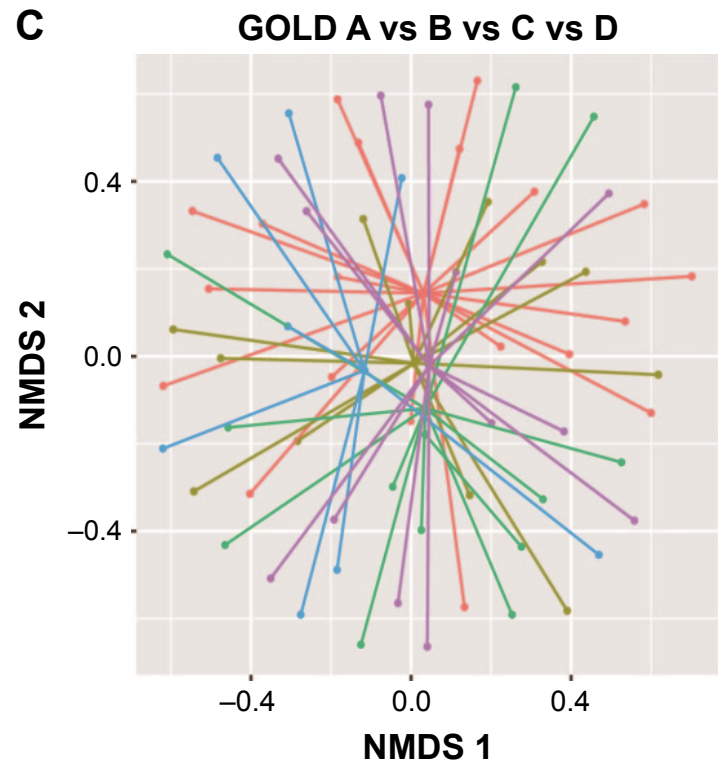

$$
\begin{aligned}
& \rightarrow \text { Control } \\
& \rightarrow-\text { GOLD_A } \\
& \rightarrow \text { GOLD_B } \\
& \rightarrow \text { GOLD_C } \\
& \rightarrow \text { GOLD_D }
\end{aligned}
$$

Jclass distance

GOLD_A-GOLD_B: $P=0.165$

GOLD_A-GOLD_C: $P=0.807$

GOLD_A-GOLD_D: $P=0.555$

GOLD_B-GOLD_C: $P=0.886$

GOLD_B-GOLD_D: $P=0.531$

GOLD_C-GOLD_D: $P=0.987$

Figure I Comparison of bacterial communities between smokers with or without COPD.

Notes: NMDS with AMOVA was conducted based on jclass distance matrix. (A) Indicated that bacterial communities between controls and $C O P D$ were different $(P=0.018$, AMOVA); (B) suggested that no difference was observed between group I and group $2(P=0.8 \mid 8$, AMOVA) and (C) implied that there is no difference between GOLD A-D groups. The COPD patients included two subgroups: group I ( $F E V_{1} \%$ pred $\geq 50 \%$ ) and group 2 (FEV \%pred $<50 \%$ ).

Abbreviations: AMOVA, analysis of molecular variance; FEV,\%pred, predicted percent of forced expiratory volume in I s; GOLD, Global Initiative on Obstructive Lung Disease; NMDS, nonmetric multidimensional scaling.

Negativicutes), orders (Lactobacillales, Selenomonadales) and families (Streptococcaceae, Veillonellaceae) were significantly different between the COPD and the control groups. The abundance of Veillonella was increased in the COPD (8.8 [4.6-13.4]) vs the control groups (4.8 [2.2-9.9]); by contrast, Streptococcus abundance was decreased in the COPD (4.8 [1.9-8.2]) vs the control groups (9.0 [5.7-17.3]).
No differentially expressed bacteria were detected between group 1 and group 2.

\section{The most abundant OTUs (species level) between COPD and controls}

Figure 5 shows an overview of the 25 most abundant OTUs (species level). A total of seven significantly different 


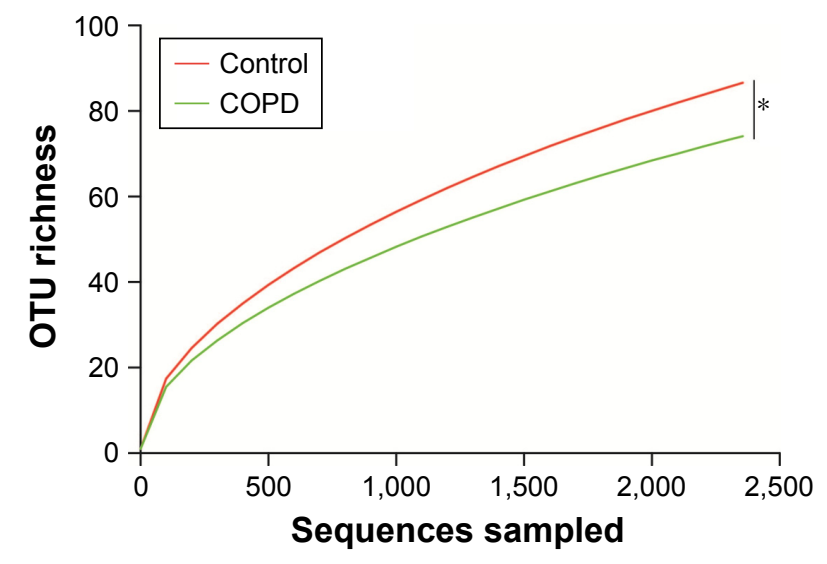

Figure 2 Rarefaction curve analyses for smokers with or without COPD. Notes: $* P<0.05$ denoted the comparison between COPD and control. Rarefaction curve indicated that OTU richness was decreased in COPD compared with that in the controls.

Abbreviations: OTU, operational taxonomic unit; COPD, chronic obstructive pulmonary disease.

OTUs were identified between the COPD and the controls. Interestingly, two highly abundant bacteria, otu3 (dispar) and otu4 (unclassified), were classified to the previously identified genera Veillonella and Streptococcus, respectively. Unfortunately, otu4 was not classified to the species level.

\section{Relationship between the throat microbiome and lung function}

To explore the relationship between different microbial taxa and lung function, Spearman correlation analyses were performed as listed in Table 2. Some taxa showed a correlation with $\mathrm{FEV}_{1}$ \%pred or RV/TLC. Otu4 (unclassified) and its corresponding father-level Bacilli (class), Lactobacillales (order), Streptococcaceae (family) and Streptococcus (genus) were positively related to $\mathrm{FEV}_{1}$ \%pred ( $r=0.391,0.354,0.352$, 0.352 and 0.352 , respectively) and negatively related to $\mathrm{RV} / \mathrm{TLC}(r=-0.263,-0.234,-0.235,-0.234$ and -0.234 , respectively). By contrast, otu3 (dispar) and its father-level Negativicutes (class), Selenomonadales (order), Veillonellaceae (family) and Veillonella (genus) were negatively correlated with $\mathrm{FEV}_{1}$ \%pred ( $r=-0.300,-0.284,-0.284,-0.282$ and -0.294 , respectively) and positively correlated with RV/ $\operatorname{TLC}(r=0.322,0.311,0.311,0.310$ and 0.321 , respectively). Several low-abundant bacteria, such as otu28 (Porphyromonas_endodontalis), were also correlated with $\mathrm{FEV}_{1}$ \%pred $(r=0.399)$ and RV/TLC $(r=-0.385)$.

\section{Assessment of bacterial taxa as a potential COPD biomarker}

To evaluate the biomarker potential of different taxa for COPD, ROC curve analyses were conducted. The area under curve (AUC) for specific bacteria was calculated based on their relative abundance. ROC curves with an AUC of greater than 0.7 are illustrated in Figure 6. No phylum-level taxa were identified. Panels (A-D) separately showed that Bacilli, Lactobacillales, Streptococcaceae and Streptococcus uniquely have the slight potential to distinguish COPD from controls (AUC $=0.707,0.706$, 0.704 and 0.704 , respectively) in their corresponding taxonomic level. And the four taxa separately represented different father levels of otu4. Figure $6 \mathrm{E}$ showed that otu4 (Streptococcaceae_unclassified), otu10 (Prevotella_ nanceiensis) and otu28 (Porphyromonas_endodontalis) could weakly identify COPD (AUC $=0.728,0.729$ and 0.736 , respectively). Combination was calculated by the relative abundance of otu 4 , otu 10 and otu 28 using logistic regression
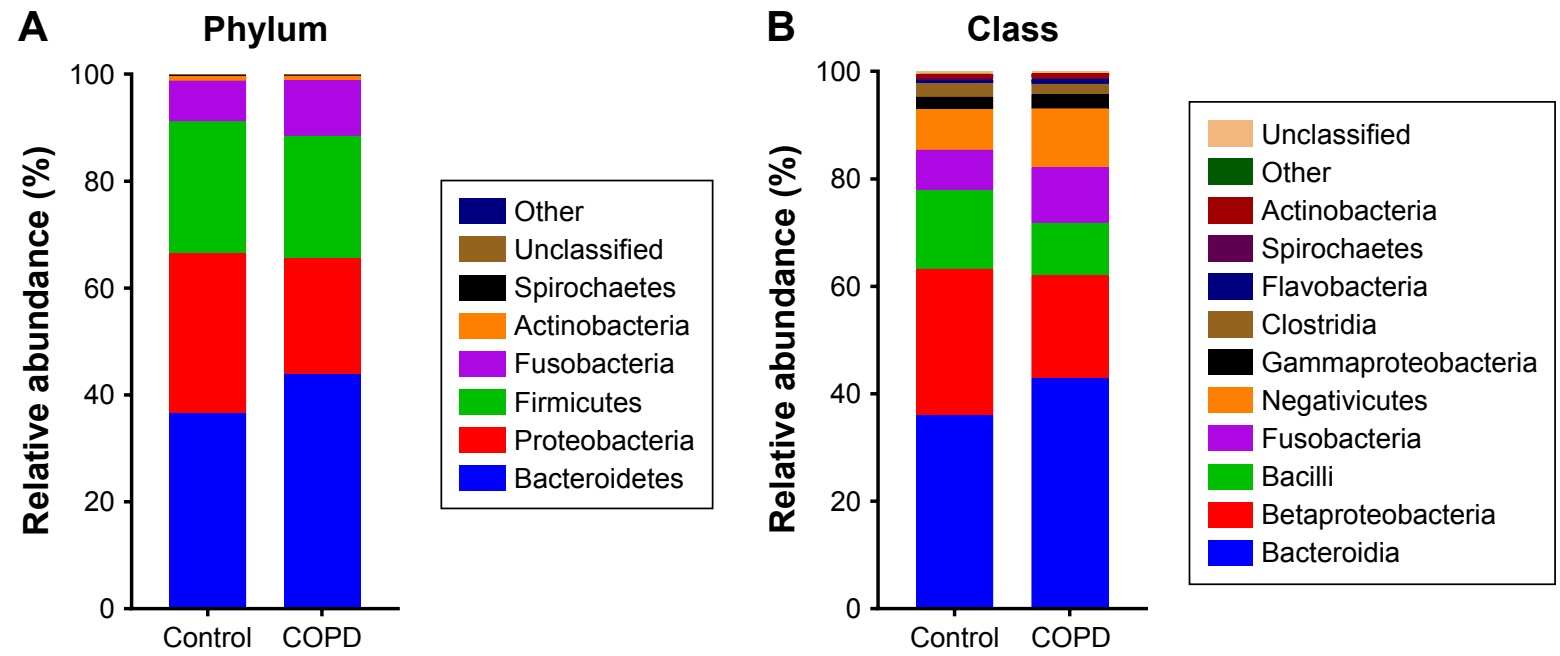

Figure 3 (Continued) 


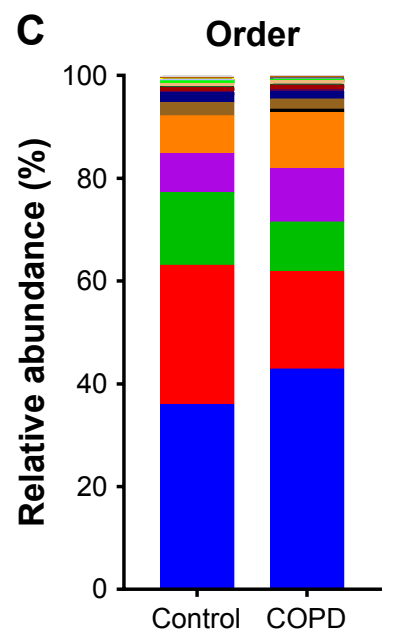

\begin{tabular}{|ll|}
\hline Unclassified & Oceanospirillales \\
Other & Pasteurellales \\
Pseudomonadales & Clostridiales \\
Coriobacteriales & Enterobacteriales \\
Alteromonadales & Selenomonadales \\
Burkholderiales & Fusobacteriales \\
Bacillales & Lactobacillales \\
Actinomycetales & Neisseriales \\
Spirochaetales & Bacteroidales \\
Flavobacteriales & \\
\hline
\end{tabular}

D

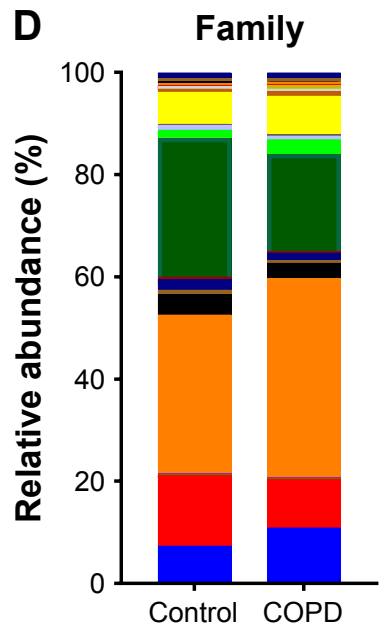

\begin{tabular}{|ll|}
\hline Unclassified & Lactobacillaceae \\
Actinomycetaceae & Leptotrichiaceae \\
Bacillales_incertae_sedis_XI & Moraxellaceae \\
Bacteroidaceae & Neisseriaceae \\
Burkholderiaceae & Other \\
Clostridiales_incertae_sedis_XI & Oxalobacteraceae \\
Coriobacteriaceae & Pasteurellaceae \\
Corynebacteriaceae & Peptostreptococcaceae \\
Enterobacteriaceae & Porphyromonadaceae \\
Eubacteriaceae & Prevotellaceae \\
Flavobacteriaceae & Veillonellaceae \\
Fusobacteriaceae & Streptococcaceae \\
Halomonadaceae & Spirochaetaceae \\
Lachnospiraceae & Shewanellaceae \\
\hline
\end{tabular}

E Genus

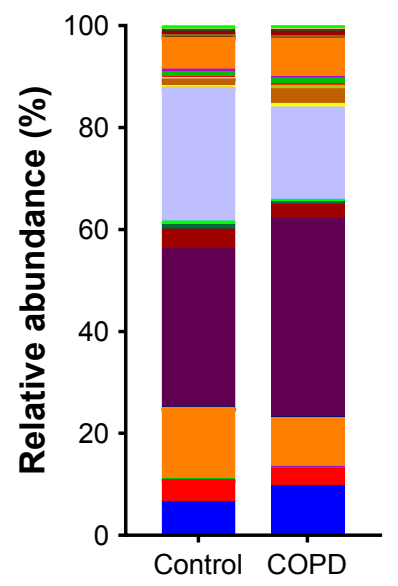

\begin{tabular}{|lll|}
\hline Actinobacillus & Haemophilus & Parvimonas \\
Actinomyces & Halomonas & Peptostreptococcus \\
Atopobium & Herbaspirillum & Porphyromonas \\
Bacteroides & Klebsiella & Prevotella \\
Capnocytophaga & Lactobacillus & Selenomonas \\
Clostridium_XIVa & Leptotrichia & Shewanella \\
Corynebacterium & Megasphaera & Tannerella \\
Eubacterium & Moraxella & Streptococcus \\
Filifactor & Neisseria & Streptobacillus \\
Fusobacterium & Oribacterium & Veillonella \\
Gemella & Other & Unclassified \\
& & Treponema \\
\hline
\end{tabular}

Figure 3 Taxonomic distribution between controls and COPD patients.

Note: Taxonomic distribution in $(\mathbf{A})$ phylum, $(\mathbf{B})$ class, $(\mathbf{C})$ order, (D) family and (E) genus level was different $(\boldsymbol{P}<0.05$, Fisher's exact test) between controls and COPD patients.

(Enter Method). However, the combination $(\mathrm{AUC}=0.746)$ did not show stronger distinct ability than otu4, otu10 and otu28 alone. More details are shown in Table S2.

\section{Discussion}

The present study aimed to investigate differences in bacterial communities between smokers with or without COPD. We found that the microbiome of COPD patients differed from that of smokers without COPD, but no differences were observed between COPD patients with different degrees of lung function or disease severity. The OTU richness decreased and the taxonomic distribution shifted with COPD. Notably, two dominant bacteria, otu3 (Veillonella_dispar), otu4 (Streptococcaceae_ unclassified), and their father-level taxa showed a correlation with lung function or biomarker potential for COPD, the alteration of which may be related to COPD pathogenesis. 


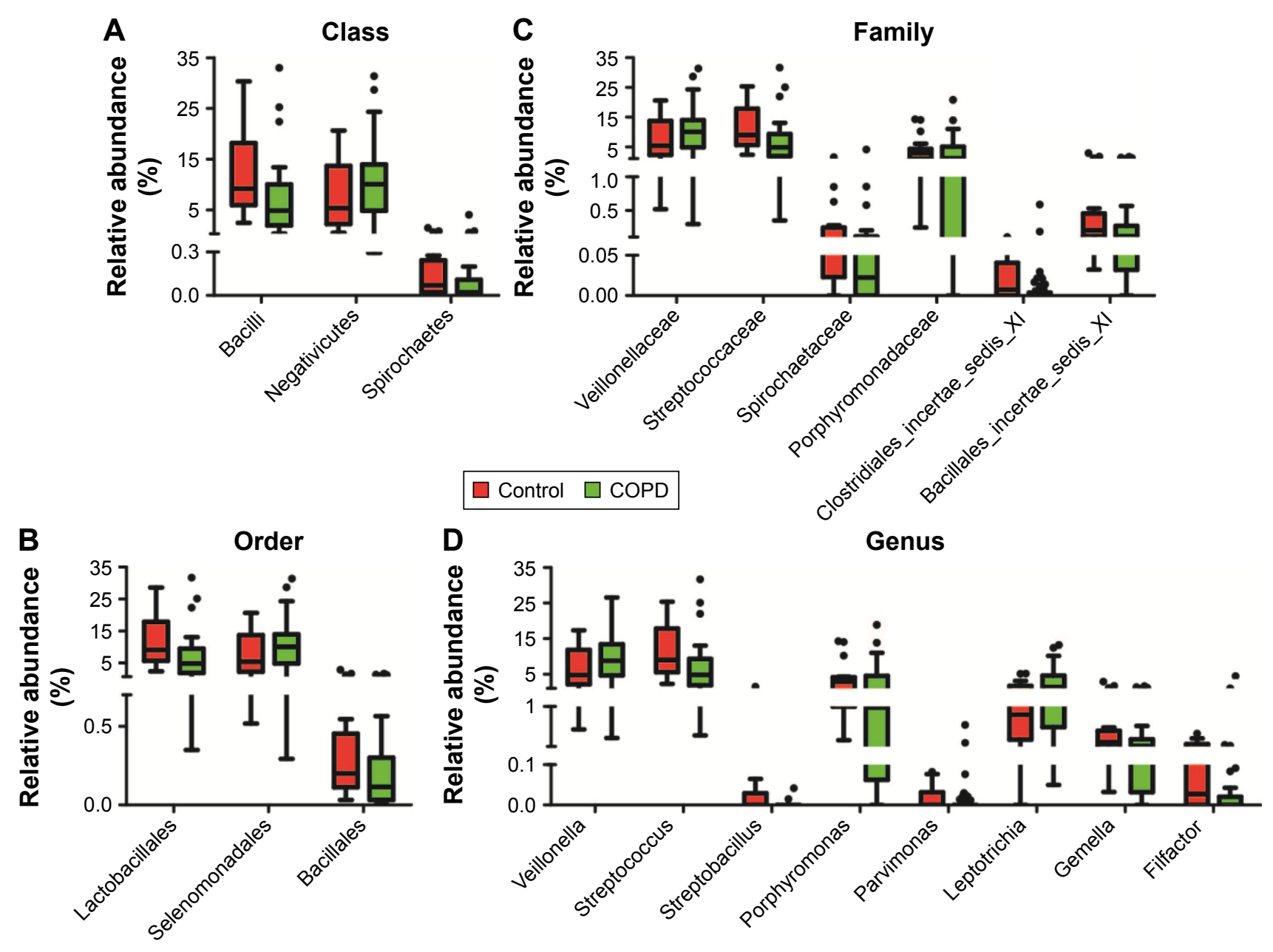

Figure 4 Prominent taxa from phylum to genus level between COPD and controls.

Notes: Prominent taxa (raw $P<0.05$ and FDR $<0.2$, Mann-Whitney $U$-test) between COPD and controls at class, order, family and genus level were plotted in panels (A-D), respectively.

Abbreviation: FDR, false discovery rate.

Results from NMDS and AMOVA suggested that the flora of the COPD groups was different from that of the controls, but there was no difference between COPD patients with different severities of lung function or disease severity, which was consistent with the results of Pragman et al from BALF and Garcia et al from sputum. ${ }^{8,17}$ Our results indicated that the OTUs, Shannon and inverse Simpson indexes showed a decreased trend in COPD, which were also similar to those of the lower airway. ${ }^{8,18}$ These findings based on overall bacterial diversity suggested that the microbiome of the throat may partly reflect alteration of the lower airway.

In the present study, the dominant phyla of the throat were Bacteroidetes, Proteobacteria, Firmicutes and Fusobacteria, which was consistent with the results from BALF and sputum samples., ${ }^{9} 19$ The dominant genera of the throat were Haemophilus, Leptotrichia, Porphyromonas, Fusobacterium, Veillonella, Streptococcus,
Neisseria and Prevotella, which were basically identical to the "bacterial core" of the lower airway., ${ }^{9,19}$ These findings demonstrated that the "core microbiome" was very similar between the upper and the lower airway, which supported Charlson et al's hypothesis that bacterial communities of lower respiratory tracts may originate from the oropharynx because of microaspiration. ${ }^{20}$

Taxonomic distribution plots showed that the dominant bacterial types between smokers with or without COPD were the same, but Fisher's exact test indicated that their proportions statistically shifted. Therefore, we attempted to identify specific taxa that altered community distribution. Two highly abundant genera, Streptococcus and Veillonella, and their corresponding classes (Bacilli, Negativicutes), orders (Lactobacillales, Selenomonadales) and families (Streptococcaceae, Veillonellaceae) showed a difference between COPD and controls. The analyses based on OTUs (species level) identified seven significant OTUs in the top 25 
A

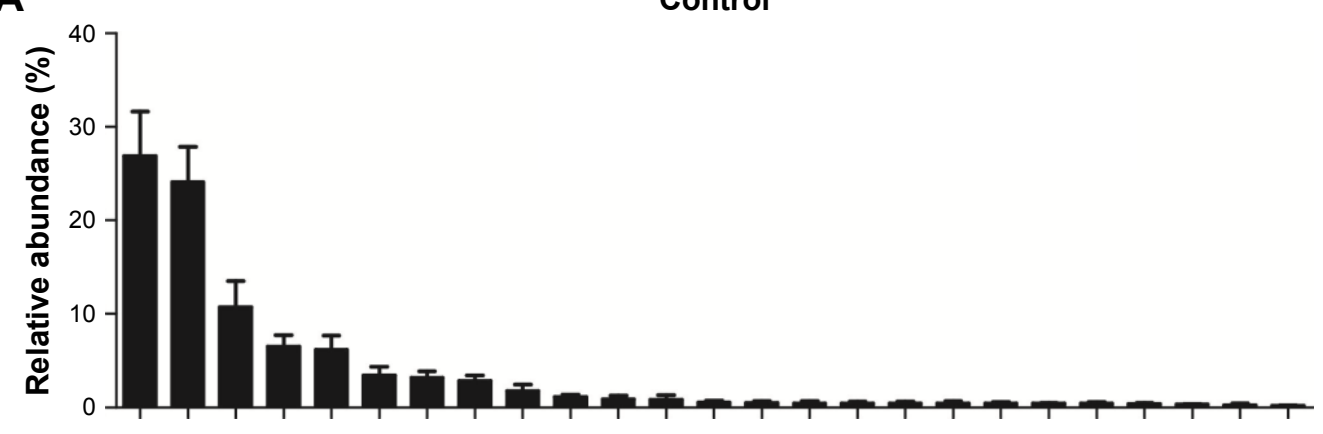

B

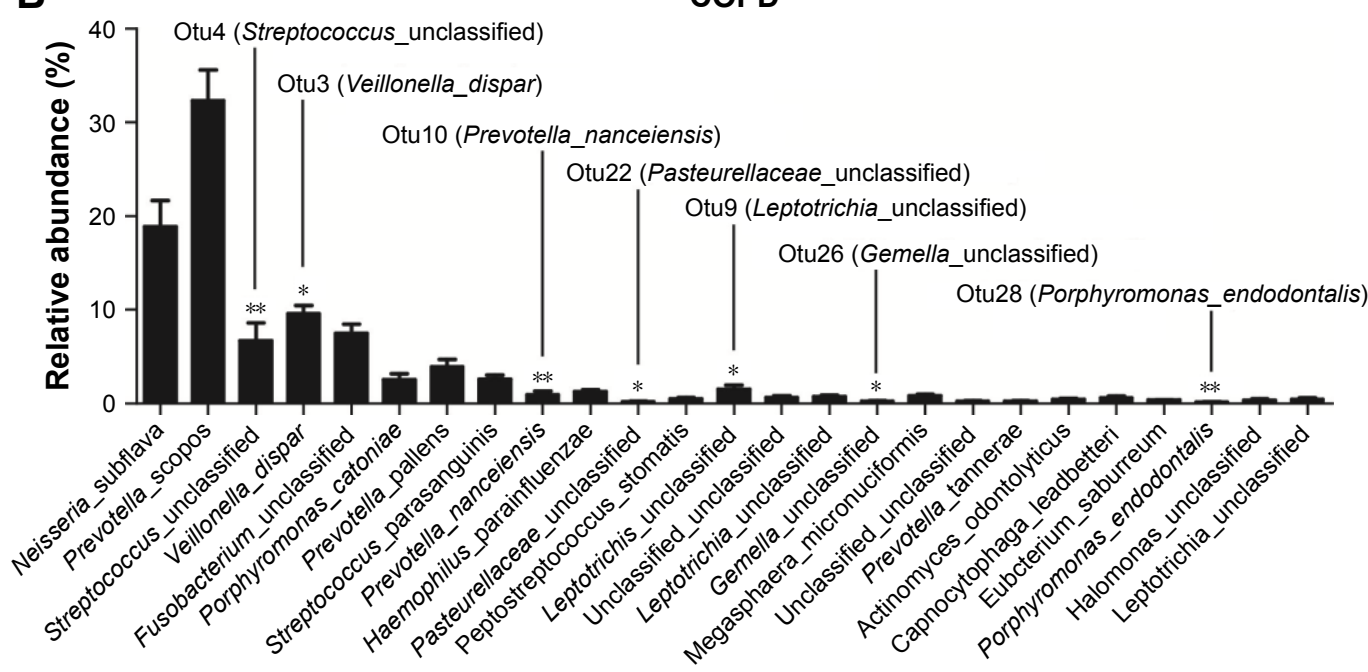

Figure 5 Relative abundance of the 25 most abundant OTUs detected in smokers with or without COPD.

Notes: The top 25 most abundant OTUs were ranked in descending order of the mean relative abundance of the controls. The seven significant OTUs (raw $P<0.05$ and FDR $<0.2$, Mann-Whitney $U$-test) are indicated.

Abbreviations: FDR, false discovery rate; OTUs, operational taxonomic units.

most abundant ones. Notably, the highly abundant otu3 (dispar) and otu4 (unclassified) were also classified to the previously identified genera Veillonella and Streptococcus, respectively. Currently, Veillonella and Streptococcus, which both belong to the phylum Firmicutes, are commonly considered part of the "core pulmonary microbiome." ",9,21 Interestingly, many studies of the lower airway and lung tissue have found that the altered abundance of bacteria from the phylum Firmicutes was related to COPD. ${ }^{17,22-24}$ Thus, we hypothesized that otu 3 and otu 4 in the throat may reflect the microbiome of the lower airway and may even be related to the pathogenesis of COPD.

The FEV ${ }_{1}$ \%pred and RV/TLC can reflect airway obstruction and emphysema, respectively, which are the most important characteristics of COPD. Thus, correlations of bacterial taxa with $\mathrm{FEV}_{1}$ \% pred and RV/TLC were calculated. Otu3 (Veillonella_dispar) and the corresponding father-level taxa, whose abundance increased in COPD, showed a negative correlation with $\mathrm{FEV}_{1} \%$ pred and a positive correlation with RV/TLC. By contrast, otu4 (Veillonella_unclassified) and the corresponding father-level taxa, whose abundance decreased in COPD, were positively correlated with $\mathrm{FEV}_{1} \%$ pred and negatively correlated with RV/TLC. These results indicated that the alteration of "core bacteria" in throat may be involved in the physiological mechanism of COPD. However, this hypothesis requires further experimental evidence.

The potential of various strains to act as a biomarker of COPD was assessed by ROC curve. We found that otu4 (Veillonella_unclassified), including its father-level taxa, otu10 (Prevotella_nanceiensis) and otu28 (Porphyromonas_endodontalis) were slightly able to distinguish COPD from controls, suggesting that throat bacteria may assist in the identification of COPD. However, the ability of these bacteria to identify COPD was weak; thus, other more prominent bacteria need to be identified in further studies.

Other bacterial taxa, such as Streptobacillus (genus level) and otu28 (Porphyromonas_endodontalis), also showed a 
Table 2 Association between bacterial taxa and lung function

\begin{tabular}{|c|c|c|c|c|}
\hline \multirow{2}{*}{$\begin{array}{l}\text { Taxonomy (\% relative } \\
\text { abundance) }\end{array}$} & \multicolumn{2}{|c|}{ FEV $\%$ pred } & \multicolumn{2}{|c|}{ RV_TLC } \\
\hline & $r$-value & $P$-value & $r$-value & $P$-value \\
\hline \multicolumn{5}{|l|}{ Phylum } \\
\hline Fusobacteria & -0.267 & 0.032 & 0.160 & 0.212 \\
\hline \multicolumn{5}{|l|}{ Class } \\
\hline Bacilli & 0.354 & 0.004 & -0.234 & 0.065 \\
\hline Fusobacteria & -0.267 & 0.032 & 0.160 & 0.212 \\
\hline Negativicutes & -0.284 & 0.022 & 0.311 & 0.013 \\
\hline \multicolumn{5}{|l|}{ Order } \\
\hline Lactobacillales & 0.352 & 0.004 & -0.235 & 0.064 \\
\hline Fusobacteriales & -0.267 & 0.032 & 0.160 & 0.212 \\
\hline Selenomonadales & -0.284 & 0.022 & 0.311 & 0.013 \\
\hline \multicolumn{5}{|l|}{ Family } \\
\hline Veillonellaceae & -0.282 & 0.023 & 0.310 & 0.013 \\
\hline Streptococcaceae & 0.352 & 0.004 & -0.234 & 0.065 \\
\hline Porphyromonadaceae & 0.268 & 0.031 & -0.268 & 0.034 \\
\hline $\begin{array}{l}\text { Clostridiales_Incertae } \\
\text { Sedis_XI }\end{array}$ & 0.313 & 0.011 & -0.297 & 0.018 \\
\hline \multicolumn{5}{|l|}{ Genus } \\
\hline Veillonella & -0.294 & 0.018 & 0.321 & 0.010 \\
\hline Streptococcus & 0.352 & 0.004 & -0.234 & 0.065 \\
\hline Streptobacillus & 0.391 & 0.001 & -0.491 & $<0.001$ \\
\hline Porphyromonas & 0.281 & 0.024 & -0.272 & 0.031 \\
\hline Parvimonas & 0.303 & 0.014 & -0.298 & 0.018 \\
\hline Filifactor & 0.328 & 0.008 & -0.356 & 0.004 \\
\hline \multicolumn{5}{|l|}{ OTU (species level) } \\
\hline otu3 (Veillonella_dispar) & -0.300 & 0.015 & 0.322 & 0.010 \\
\hline $\begin{array}{l}\text { otu4 (Streptococcus_ } \\
\text { unclassified) }\end{array}$ & 0.391 & 0.001 & -0.263 & 0.037 \\
\hline $\begin{array}{l}\text { otu7 (Porphyromonas_ } \\
\text { catoniae) }\end{array}$ & 0.245 & 0.049 & -0.236 & 0.063 \\
\hline $\begin{array}{l}\text { otu } 10 \text { (Prevotella } \\
\text { nanceiensis) }\end{array}$ & 0.337 & 0.006 & -0.218 & 0.086 \\
\hline $\begin{array}{l}\text { otu22 (Pasteurellaceae } \\
\text { unclassified) }\end{array}$ & 0.318 & 0.010 & -0.240 & 0.058 \\
\hline $\begin{array}{l}\text { otu28 (Porphyromonas_ } \\
\text { endodontalis) }\end{array}$ & 0.399 & 0.001 & -0.385 & 0.002 \\
\hline
\end{tabular}

Note: Correlation of bacterial taxa with FEV,\%pred and RV/TLC was evaluated by Spearman coefficient.

Abbreviations: OTU, operational taxonomic unit; FEV,\%pred, predicted percent of forced expiratory volume in I s; RV, residual capacity; TLC, total lung capacity.

A

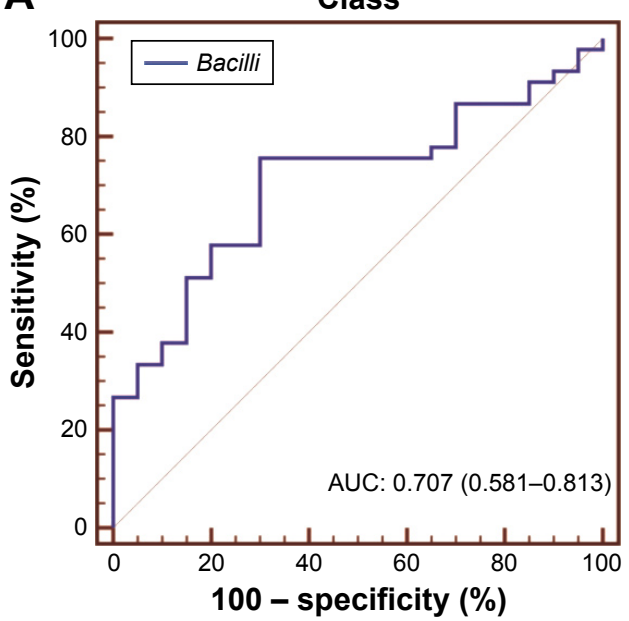

significant difference, correlation or biomarker potential in COPD compared with that of the controls. However, these results need to be confirmed in a larger population because of their low abundance.

Potential factors influencing throat microbiome were acquired and analyzed. No special diet habits, known periodontal disease and recent systematic antibiotics were reported. Antiseptic mouthwash, bronchodilators and ICS were not also used in $12 \mathrm{~h}$ before sample collection. Only long-standing ICS was significantly different between COPD patients with differing severity. However, NMDS and AMOVA implied that throat flora were very similar between group 1 and group 2 or different GOLD stages. Therefore, the impact is expected to be negligible.

Our study has the following strengths. First, compared with previous pilot studies of oropharynx microbiome, ${ }^{11,20}$ the present study included more COPD patients. Second, only smokers were included in this study, excluding the effect of smoking on oral flora. ${ }^{22}$

There were several limitations in the present study. First, no female subjects were enrolled because COPD patients in China are almost exclusively males; second, follow-up of the population has not been conducted; third, samples from the lower respiratory tracts were not collected; Fourth, the rarefaction curves still showed an increasing trend, suggesting that sequencing depth was still not enough. Thus, it is necessary that a newer sequencing technique such as Miseq is used in future; Fifth, the present cross-sectional trial was still a small sample-size study, restricting the further analyses. In future, it is necessary to determine the relationship between the throat microbiome and the progression of COPD in a larger cohort with a long-term follow-up.
B

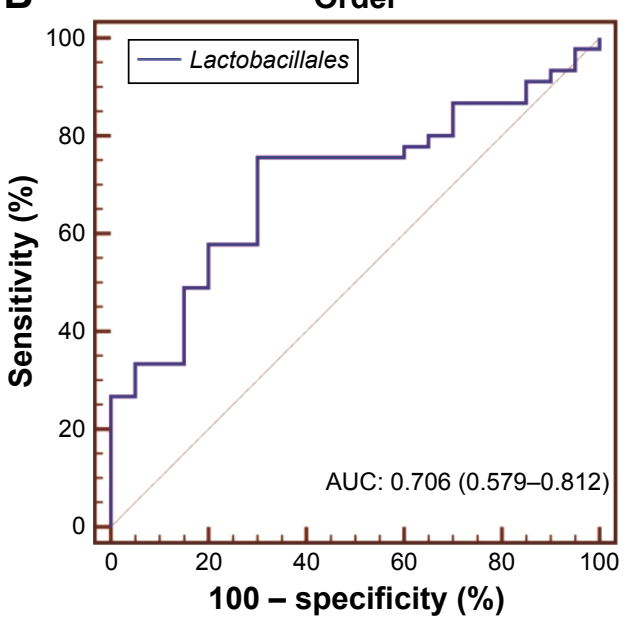

Figure 6 (Continued) 

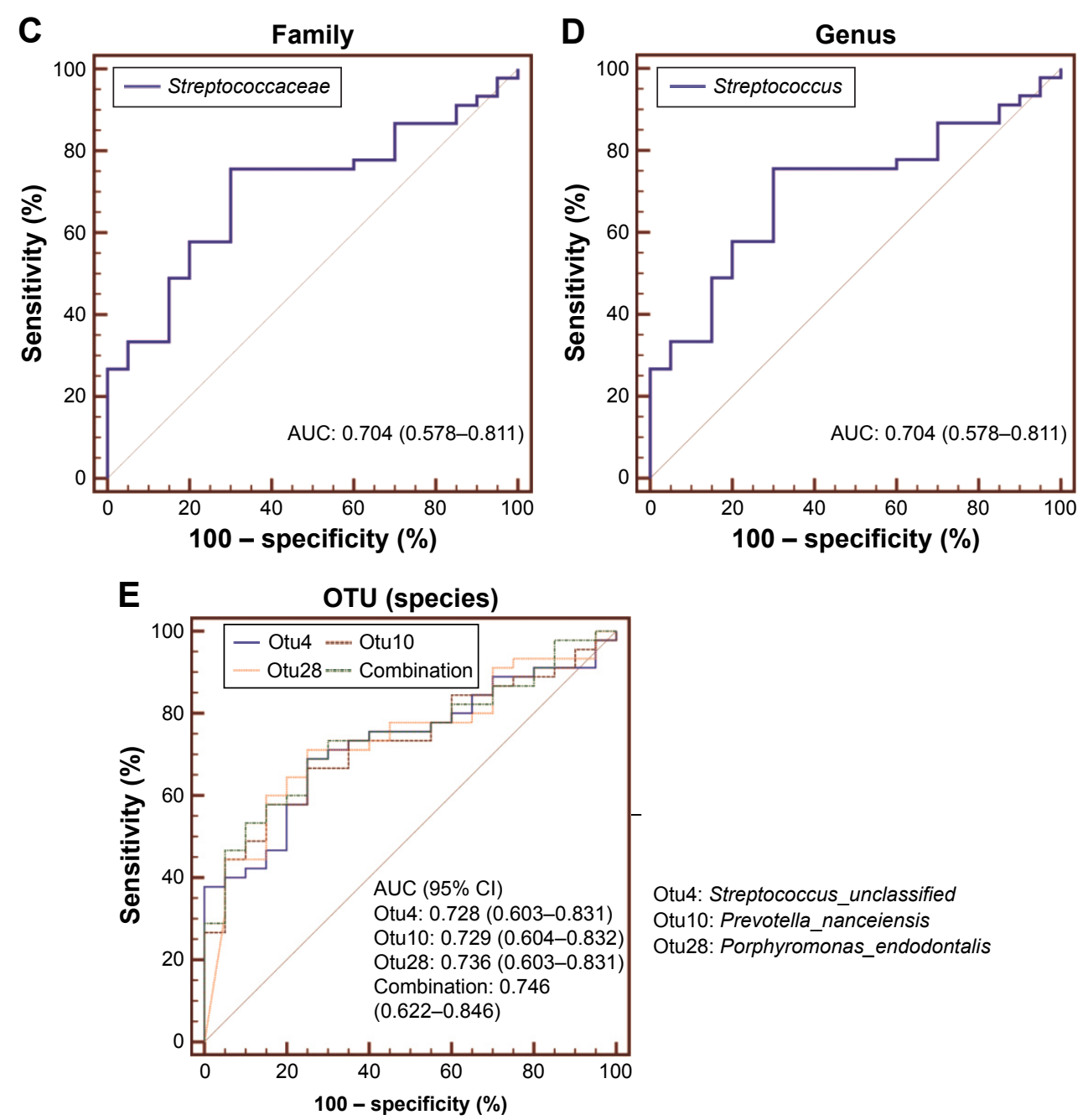

Otu4: Streptococcus_unclassified

Otu10: Prevotella_nanceiensis

Otu28: Porphyromonas_endodontalis

Figure 6 Biomarker potential for COPD at different taxonomic levels.

Notes: ROC curve analysis was used to evaluate the biomarker potential of specific taxa in (A) class, (B) order, (C) family, (D) genus and (E) OTU (species) level for COPD. Bacterial taxa with an AUC of greater than 0.7 are presented.

Abbreviations: AUC, area under curve; OTU, operational taxonomic unit; ROC, receiver operating characteristic.

\section{Conclusion}

In summary, the present study found that the throat microbiome differed among smokers with or without COPD, and the results are similar to those from lower respiratory tracts. These data can help us to better understand the relationship between the microbiome of different airway sites and pathogenesis of COPD.

\section{Acknowledgments}

The present study was funded by the National Natural Science Foundation of China (Grant Nos: 81270097; 81470235) and BMU (Grant No: 20110176). The funders had no role in study design, data collection and analysis, decision to publish, or preparation of the manuscript.

\section{Author contributions}

All authors declared their personal contribution to this manuscript: BH conducted study design and crucially revised the article; WD conducted bioinformatic analyses and biological interpretation, and wrote the manuscript; NS was involved in statistical analyses and enrolled subjects; YD collected throat samples and performed lung function test for subjects; KQ gathered the clinical information of subjects. All authors contributed toward data analysis, drafting and critically revising the paper, approved the final version of the manuscript and agreed to be accountable for all aspects of the work.

\section{Disclosure}

The authors report no conflicts of interest in this work. 


\section{References}

1. Barnes PJ, Celli BR. Systemic manifestations and comorbidities of COPD. Eur Respir J. 2009;33(5):1165-1185.

2. Patel IS, Vlahos I, Wilkinson TM, et al. Bronchiectasis, exacerbation indices, and inflammation in chronic obstructive pulmonary disease. Am J Respir Crit Care Med. 2004;170(4):400-407.

3. Papi A, Bellettato CM, Braccioni F, et al. Infections and airway inflammation in chronic obstructive pulmonary disease severe exacerbations. Am J Respir Crit Care Med. 2006;173(10):1114-1121.

4. Rosell A, Monso E, Soler N, et al. Microbiologic determinants of exacerbation in chronic obstructive pulmonary disease. Archiv Intern Med. 2005;165(8):891-897.

5. Lee SW, Kuan CS, Wu LS, Weng JT. Metagenome and metatranscriptome profiling of moderate and severe COPD sputum in Taiwanese Han males. PLoS One. 2016;11(7):e0159066.

6. Huang YJ, Erb-Downward JR, Dickson RP, Curtis JL, Huffnagle GB, Han MK. Understanding the role of the microbiome in chronic obstructive pulmonary disease: principles, challenges, and future directions. Transl Res. 2017;179:71-83.

7. Wang Z, Bafadhel M, Haldar K, et al. Lung microbiome dynamics in COPD exacerbations. Eur Respir J. 2016;47(4):1082-1092.

8. Garcia-Nunez M, Millares L, Pomares X, et al. Severity-related changes of bronchial microbiome in chronic obstructive pulmonary disease. J Clin Microbiol. 2014;52(12):4217-4223.

9. Erb-Downward JR, Thompson DL, Han MK, et al. Analysis of the lung microbiome in the "healthy" smoker and in COPD. PLoS One. 2011; 6(2):e16384

10. Ogorodova LM, Fedosenko SV, Popenko AS, et al. Comparison study of oropharyngeal microbiota in case of bronchial asthma and chronic obstructive pulmonary disease in different severity levels. Vestn Ross Akad Med Nauk. 2015;(6):669-678. Russian.

11. Park H, Shin JW, Park SG, Kim W. Microbial communities in the upper respiratory tract of patients with asthma and chronic obstructive pulmonary disease. PLoS One. 2014;9(10):e109710.

12. Vestbo J, Hurd SS, Agusti AG, et al. Global strategy for the diagnosis, management, and prevention of chronic obstructive pulmonary disease: GOLD executive summary. Am J Respir Crit Care Med. 2013; 187(4):347-365.
13. Mason KL, Erb Downward JR, Mason KD, et al. Candida albicans and bacterial microbiota interactions in the cecum during recolonization following broad-spectrum antibiotic therapy. Infect Immun. 2012; 80(10):3371-3380.

14. Jumpstart Consortium Human Microbiome Project Data Generation Working Group. Evaluation of 16S rDNA-based community profiling for human microbiome research. PLoS One. 2012;7(6):e39315.

15. Hov JR, Zhong H, Qin B, et al. The influence of the autoimmunityassociated ancestral HLA haplotype AH8.1 on the human gut microbiota: a cross-sectional study. PLoS One. 2015;10(7):e0133804.

16. Schloss PD, Westcott SL, Ryabin T, et al. Introducing mothur: open-source, platform-independent, community-supported software for describing and comparing microbial communities. Appl Environ Microbiol. 2009;75(23):7537-7541.

17. Pragman AA, Kim HB, Reilly CS, Wendt C, Isaacson RE. The lung microbiome in moderate and severe chronic obstructive pulmonary disease. PLoS One. 2012;7(10):e47305.

18. Einarsson GG, Comer DM, McIlreavey L, et al. Community dynamics and the lower airway microbiota in stable chronic obstructive pulmonary disease, smokers and healthy non-smokers. Thorax. 2016; 71(9):795-803.

19. Galiana A, Aguirre E, Rodriguez JC, et al. Sputum microbiota in moderate versus severe patients with COPD. Eur Respir J. 2014; 43(6):1787-1790.

20. Charlson ES, Bittinger K, Haas AR, et al. Topographical continuity of bacterial populations in the healthy human respiratory tract. Am J Respir Crit Care Med. 2011;184(8):957-963.

21. Zakharkina T, Heinzel E, Koczulla RA, et al. Analysis of the airway microbiota of healthy individuals and patients with chronic obstructive pulmonary disease by T-RFLP and clone sequencing. PLoS One. 2013;8(7):e68302.

22. Hilty M, Burke C, Pedro H, et al. Disordered microbial communities in asthmatic airways. PLoS One. 2010;5(1):e8578.

23. Huang YJ, Kim E, Cox MJ, et al. A persistent and diverse airway microbiota present during chronic obstructive pulmonary disease exacerbations. Omics. 2010;14(1):9-59.

24. Sze MA, Dimitriu PA, Hayashi S, et al. The lung tissue microbiome in chronic obstructive pulmonary disease. Am J Respir Crit Care Med. 2012;185(10):1073-1080. 


\section{Supplementary materials}

A

Control vs COPD

Bray-Curtis distance Con-COPD: $P=0.042$

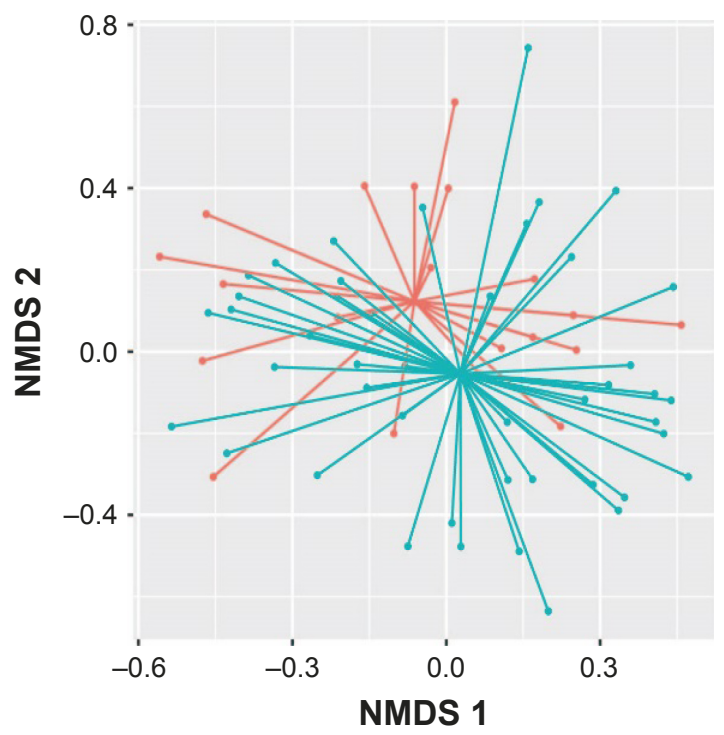

Control - COPD
B

\section{Group 1 and group 2}

Bray-Curtis distance

Group 1-group 2: $P=0.961$

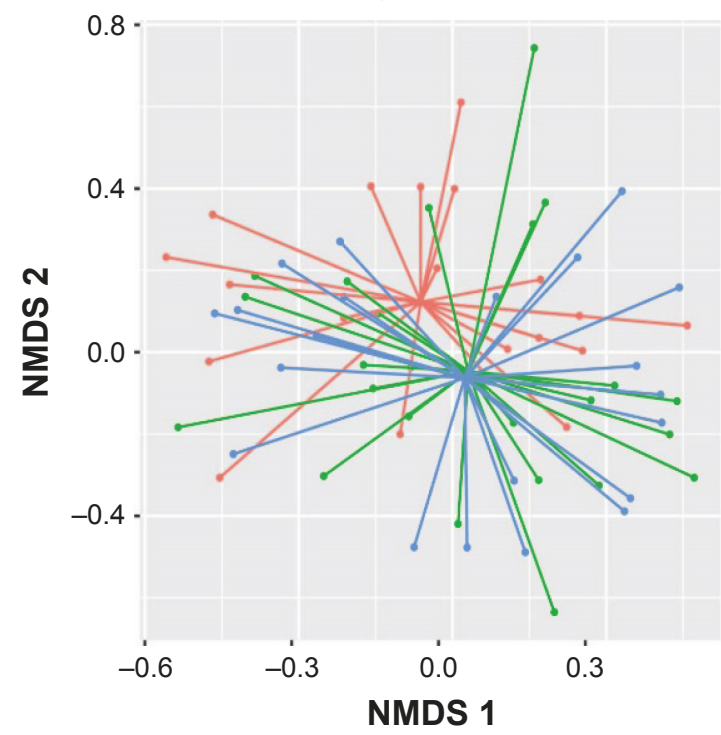

- Control

- Group 1 (FEV $\%$ pred $\geq 50 \%$ )

- Group 2 (FEV $\%$ pred <50\%)

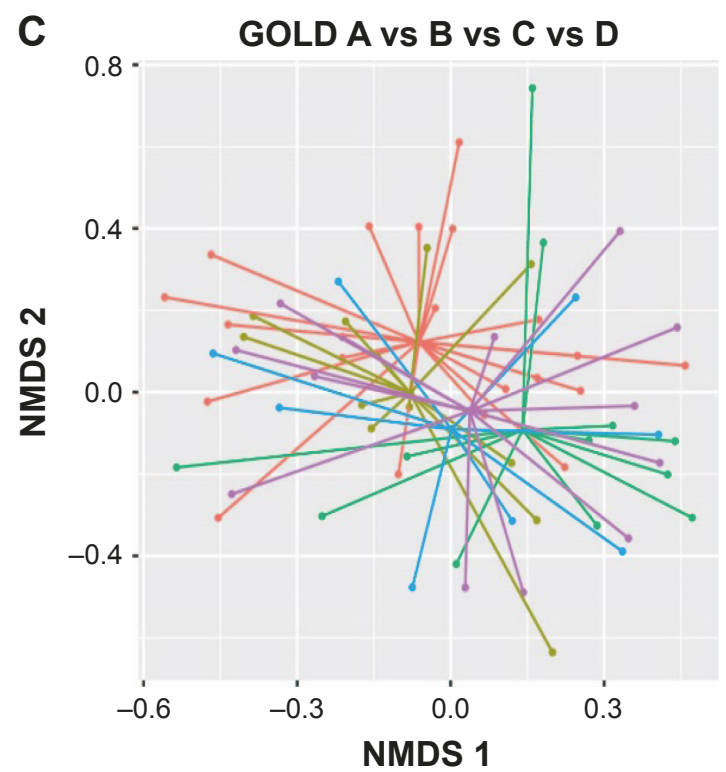

$$
\begin{aligned}
& \rightarrow \text { Control } \\
& \rightarrow \text { GOLD_A } \\
& \rightarrow \text { GOLD_B } \\
& \rightarrow \text { GOLD_C } \\
& - \text { GOLD_D }
\end{aligned}
$$

Bray-Curtis distance

GOLD_A-GOLD_B: $P=0.053$

GOLD_A-GOLD_C: $P=0.522$

GOLD_A-GOLD_D: $P=0.641$

GOLD_B-GOLD_C: $P=0.697$

GOLD_B-GOLD_D: $P=0.412$

GOLD_C-GOLD_D: $P=0.896$

Figure SI Community difference (Bray-Curtis distance) between controls and COPD.

Notes: NMDS with AMOVA was conducted based on Bray-Curtis distance matrix. (A) Indicated that bacterial communities between controls and COPD were different $(P=0.042$, AMOVA); (B) suggested that no difference was observed between group I and group 2 ( $P=0.96 \mathrm{I}$, AMOVA); and (C) implied that there is no difference between GOLD A-D group. The COPD patients included two subgroups: group I (FEV,\%pred $\geq 50 \%$ ) and group 2 (FEV, \%pred $<50 \%$ ).

Abbreviations: AMOVA, analysis of molecular variance; GOLD, Global Initiative on Obstructive Lung Disease; FEV , forced expiratory volume in I s; NMDS, nonmetric multidimensional scaling. 
A

Group 1 vs group 2

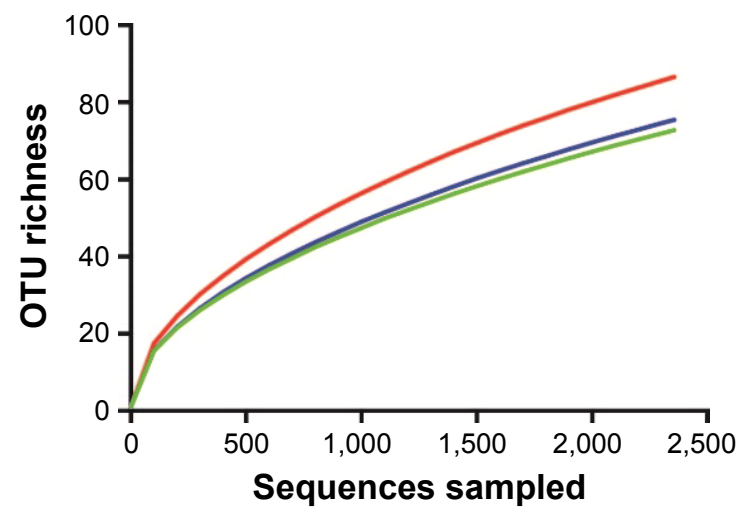

- Control - Group 1 - Group 2
B GOLD A vs B vs C vs D

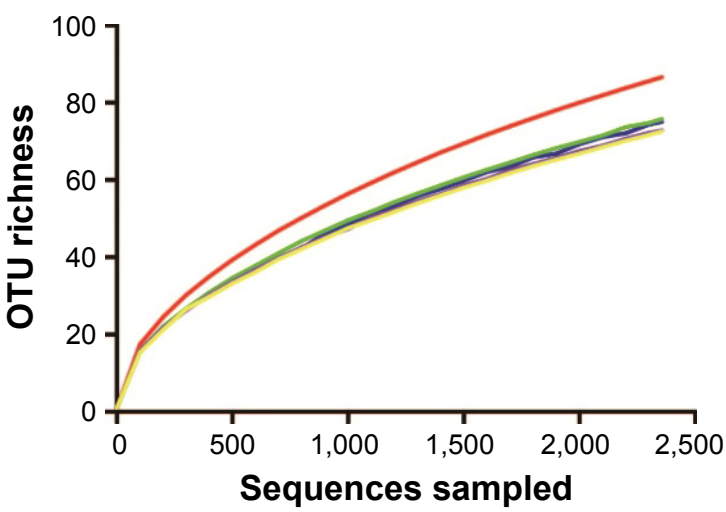

Figure S2 Rarefaction curve analyses for COPD with different degrees of lung function or disease severity.

Notes: (A) The COPD patients included two subgroups: group I (FEV \%pred $\geq 50 \%$ ); group 2 (FEV \%pred $<50 \%$ ). No shift was observed between group I and group 2 , and (B) no difference was identified between different GOLD classification.

Abbreviation: GOLD, Global Initiative on Obstructive Lung Disease.
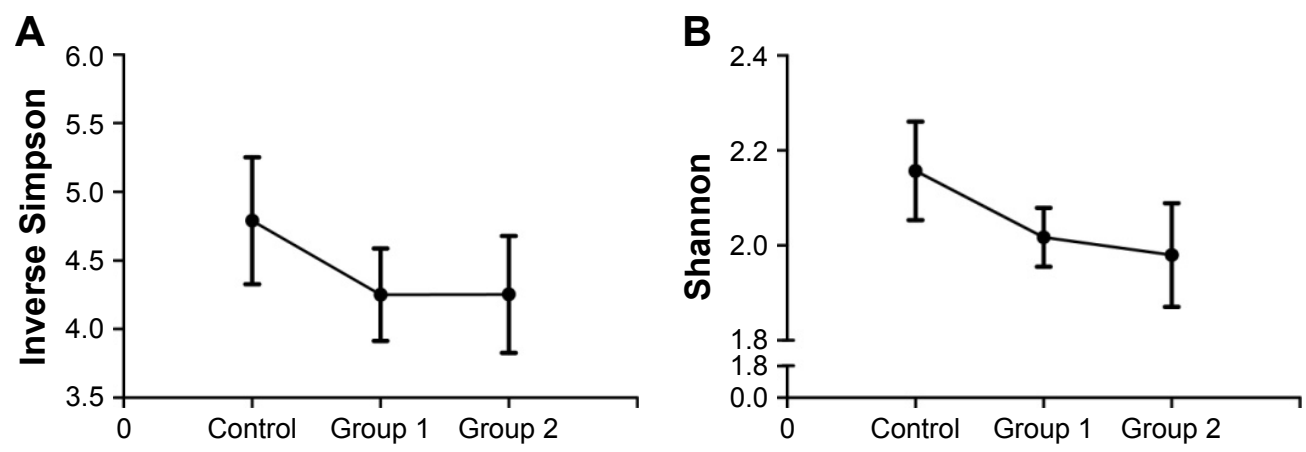

Figure S3 Comparison of Shannon and inverse Simpson index among different groups.

Notes: Inverse Simpson $(\mathbf{A})$ and Shannon $(\mathbf{B})$ index showed a decreasing trend in COPD patients than controls but no observed difference between group I and group 2. COPD patients included two subgroups: group I (FEV,\%pred $\geq 50 \%$ ) and group 2 ( $\mathrm{FEV}, \%$ pred $<50 \%$ ).

Table SI Significant taxa between COPD and controls at different taxonomic levels

\begin{tabular}{|c|c|c|c|c|}
\hline Significant taxa* & Control & COPD & Raw P-value & FDR \\
\hline \multicolumn{5}{|l|}{ Class } \\
\hline Bacilli & $9.20(6.02-17.68)$ & $4.87(1.96-8.53)$ & 0.008 & 0.091 \\
\hline Negativicutes & $5.38(2.26-10.75)$ & $10.07(4.90-13.89)$ & 0.027 & 0.163 \\
\hline Spirochaetes & $0.07(0.04-0.23)$ & $0.02(0.00-0.11)$ & 0.050 & 0.19 \\
\hline \multicolumn{5}{|l|}{ Order } \\
\hline Lactobacillales & $9.03(5.80-17.43)$ & $4.85(I .9 I-8.4 I)$ & 0.008 & 0.151 \\
\hline Selenomonadales & $5.38(2.26-10.75)$ & $10.07(4.90-13.89)$ & 0.027 & 0.173 \\
\hline Bacillales & $0.20(0.12-0.38)$ & $0.11(0.03-0.29)$ & 0.024 & 0.173 \\
\hline
\end{tabular}


Table SI (Continued)

\begin{tabular}{|c|c|c|c|c|}
\hline Significant taxa* & Control & COPD & Raw P-value & FDR \\
\hline \multicolumn{5}{|l|}{ Family } \\
\hline Veillonellaceae & $5.38(2.26-10.75)$ & $10.07(4.90-13.86)$ & 0.028 & 0.171 \\
\hline Streptococcaceae & 9.01 (5.79-17.40) & $4.83(1.91-8.25)$ & 0.008 & 0.171 \\
\hline Spirochaetaceae & $0.07(0.04-0.23)$ & $0.02(0.00-0.11)$ & 0.050 & 0.177 \\
\hline Porphyromonadaceae & $2.91(1.28-4.19)$ & $1.07(0.08-4.89)$ & 0.043 & 0.171 \\
\hline Clostridiales_Incertae_Sedis_XI & $0.01(0.00-0.04)$ & $0.00(0.00-0.00)$ & 0.026 & 0.171 \\
\hline Bacillales_Incertae_Sedis_XI & $0.20(0.12-0.38)$ & $0.11(0.03-0.24)$ & 0.022 & 0.171 \\
\hline \multicolumn{5}{|l|}{ Genus } \\
\hline Veillonella & $4.78(2.19-9.95)$ & $8.76(4.64-13.43)$ & 0.030 & 0.163 \\
\hline Streptococcus & 9.01 (5.79-17.39) & $4.83(1.91-8.25)$ & 0.008 & 0.094 \\
\hline Streptobacillus & $0.00(0.00-0.03)$ & $0.00(0.00-0.00)$ & 0.001 & 0.031 \\
\hline Porphyromonas & $2.87(1.22-4.05)$ & $0.98(0.06-4.23)$ & 0.031 & 0.163 \\
\hline Parvimonas & $0.00(0.00-0.03)$ & $0.00(0.00-0.00)$ & 0.048 & 0.197 \\
\hline Leptotrichia & $0.82(0.36-1.26)$ & $1.34(0.59-4.05)$ & 0.034 & 0.163 \\
\hline Gemella & $0.20(0.12-0.38)$ & $0.11(0.03-0.24)$ & 0.022 & 0.163 \\
\hline Filifactor & $0.03(0.00-0.14)$ & $0.00(0.00-0.02)$ & 0.003 & 0.051 \\
\hline \multicolumn{5}{|l|}{ OTU (species level) } \\
\hline otu3 (Veillonella_dispar) & $4.73(2.10-9.89)$ & $8.52(4.63-13.10)$ & 0.035 & 0.176 \\
\hline otu4 (Streptococcus_unclassified) & $6.02(4.77-10.99)$ & $2.46(0.70-5.83)$ & 0.003 & 0.034 \\
\hline otu9 (Leptotrichia_unclassified) & $0.12(0.03-0.57)$ & $0.54(0.13-0.99)$ & 0.041 & 0.177 \\
\hline otul0 (Prevotella_nanceiensis) & $0.73(0.43-1.58)$ & $0.16(0.02-0.78)$ & 0.003 & 0.034 \\
\hline otu22 (Pasteurellaceae_unclassified) & $0.07(0.02-0.57)$ & $0.01(0.00-0.09)$ & 0.025 & 0.153 \\
\hline otu26 (Gemella_unclassified) & $0.20(0.13-0.38)$ & $0.11(0.03-0.24)$ & 0.023 & 0.153 \\
\hline otu28 (Porphyromonas_endodontalis) & $0.23(0.12-0.47)$ & $0.06(0.00-0.21)$ & 0.002 & 0.034 \\
\hline
\end{tabular}

Notes: *Significant taxa: both raw $P<0.05$ calculated by Mann-Whitney $U$-test and FDR $<0.2$. Value of relative abundance was represented via "median (interquartile range)." Abbreviations: FDR, false discovery rate; OTU, operational taxonomic unit.

Table S2 Area under curve, sensitivity, and specificity of different taxonomy

\begin{tabular}{|c|c|c|c|}
\hline Taxonomy & AUC $(95 \% \mathrm{Cl})$ & Sensitivity (\%) & Specificity (\%) \\
\hline \multicolumn{4}{|l|}{ Class } \\
\hline Bacilli & $0.707(0.58 \mathrm{I}-0.8 \mathrm{I} 3)$ & 75.6 & 70.0 \\
\hline \multicolumn{4}{|l|}{ Order } \\
\hline Lactobacillales & $0.706(0.579-0.812)$ & 75.6 & 70.0 \\
\hline \multicolumn{4}{|l|}{ Family } \\
\hline Streptococcaceae & $0.704(0.578-0.81 \mathrm{I})$ & 75.6 & 70.0 \\
\hline \multicolumn{4}{|l|}{ Genus } \\
\hline Streptococcus & $0.704(0.578-0.8 \mathrm{II})$ & 75.6 & 70.0 \\
\hline \multicolumn{4}{|l|}{ OTU } \\
\hline otu4 (Streptococcus_unclassified) & $0.728(0.603-0.83 I)$ & 68.9 & 75.0 \\
\hline otu 10 (Prevotella_nanceiensis) & $0.729(0.604-0.832)$ & 57.8 & 85.0 \\
\hline otu28 (Porphyromonas_endodontalis) & $0.736(0.603-0.83 I)$ & 71.1 & 75.0 \\
\hline Combination & $0.746(0.622-0.846)$ & 68.9 & 75.0 \\
\hline
\end{tabular}

Abbreviations: AUC, area under curve; $\mathrm{Cl}$, confidence interval; OTU, operational taxonomic unit.

\section{Publish your work in this journal}

The International Journal of COPD is an international, peer-reviewed journal of therapeutics and pharmacology focusing on concise rapid reporting of clinical studies and reviews in COPD. Special focus is given to the pathophysiological processes underlying the disease, intervention programs, patient focused education, and self management protocols.

\section{Dovepress}

This journal is indexed on PubMed Central, MedLine and CAS. The manuscript management system is completely online and includes a very quick and fair peer-review system, which is all easy to use. Visit http://www.dovepress.com/testimonials.php to read real quotes from published authors. 